\title{
Effect of Lo Han Kuo (Siraitia grosvenori Swingle) on Nasal Rubbing and Scratching Behavior in ICR Mice
}

\author{
Maria Alejandra Hossen, ${ }^{a}$ Yoshifumi Shinmei, ${ }^{a}$ Shuishi Jiang, ${ }^{a}$ Miho Takubo, ${ }^{a}$ Tae Tsumuro, ${ }^{a}$ \\ Yuji Murata, ${ }^{b}$ Masaki Sugiura, ${ }^{b}$ and Chiaki KameI ${ }^{*, a}$ \\ ${ }^{a}$ Department of Pharmacology, Faculty of Pharmaceutical Sciences, Okayama University; Okayama 700-8530, Japan: \\ and ${ }^{b}$ Biochemical Laboratory, Saraya Co. Ltd.; 24-12 Tomata-cho, Kashiwara, Osaka 582-0028, Japan. \\ Received August 19, 2004; accepted October 29, 2004
}

We studied the effect of Lo Han Kuo (Siraitia grosvenori Swingle) on histamine-induced nasal rubbing and compound 48/80-induced skin scratching behavior in ICR mice. An extract and glycoside (a complex of sweet components) of Lo Han Kuo were used in the study. Both the extract and glycoside caused no significant effect on nasal rubbing or scratching behavior, even at a dose of $1000 \mathrm{mg} / \mathrm{kg}$ when administered in a single dose. However, the effect of Lo Han Kuo became clear after repeated administration, and 300 and $1000 \mathrm{mg} / \mathrm{kg}$ of both extract and glycoside significantly inhibited nasal rubbing and skin scratching behavior after consecutive treatment for 4 weeks. Both the extract and glycoside inhibited the histamine release induced by compound $48 / 80$ at concentrations of 300 and $1000 \mu \mathrm{g} / \mathrm{ml}$. From these results, it is assumed that the inhibition of nasal rubbing and skin scratching behavior induced by Lo Han Kuo occurs through a mast cell-dependent mechanism.

Key words Lo Han Kuo; histamine; compound 48/80; nasal rubbing; scratching behavior; histamine release

Lo Han Kuo (Siraitia grosvenori Swingle) is the fruit of the Siraitia grosvenori plant. ${ }^{1)}$ This plant has been cultivated in a restricted area of the southern part of China, Guangxi Province, and only the heated and dried fruit or extract were traded to other countries such as southeast Asia, Japan and the United States of America. ${ }^{2)}$ Lo Han Kuo has been used as a sweet beverage, it contains sweet components called mogrosides, which are triterpene glycosides 400 times sweeter than sucrose. ${ }^{3)}$ In addition, the extract of the dried fruit has been employed as a folk medicine for pharyngitis, cough and stomach or intestinal catarrh. ${ }^{4}$ Konoshima and Takasaki $^{2}$ found that the mogroside $\mathrm{V}$ contained in Lo Han Kuo might be valuable as a chemopreventive agent against chemical carcinogenesis. It has been recognized that the overproduction of nitric oxide, or NO radicals, induce mutation of genes and strongly initiate multistage carcinogenesis. $^{5,6)}$ When Siratia grosvenori extract was tested on lowdensity lipoprotein (LDL) oxidation, it was found that its sweet elements reduced the atherogenic potential of LDL by inhibiting not only copper-mediated, but also human umbilical vein endothelial cell-mediated LDL oxidation in a dosedependent manner. ${ }^{7)}$ Similarly, Shi et al. demonstrated that Lo Han Kuo extract has antioxidant activity against free radicals generated by a hypoxanthine and xanthine oxidase system and $\mathrm{Fe}(\mathrm{II})$-induced lipid peroxidation. ${ }^{8)}$ It is well established that drugs having an antioxidant effect have anti-allergic effects as well, and that superoxide generation plays an important role in mast cell activation. ${ }^{9-11)}$ The release of chemical mediators from tissue mast cells has been centrally implicated in a diversity of allergic and inflammatory disorders. Therefore, in the present study, the effect of Lo Han Kuo on allergic symptoms such as nasal rubbing and skin scratching behavior induced by histamine and compound 48/80, respectively, were studied in ICR mice.

\section{MATERIALS AND METHODS}

Animals Female ICR mice (6-10 week-old) were obtained from Japan SLC, Inc., Shizuoka. The animals were housed in an air-conditioned room maintained at $24 \pm 2{ }^{\circ} \mathrm{C}$ with a relative humidity of $55 \pm 15 \%$. They were given standard laboratory rodent chow (Oriental Yeast, Tokyo) and water ad libitum. All procedures involving animals were conducted in accordance with the guidelines of the Animal Care and Use Committee, Faculty of Pharmaceutical Sciences, Okayama University.

Lo Han Kuo Samples To obtain Lo Han Kuo extract, fresh Lo Han Kuo fruits was washed and crushed; then, an extraction was done using hot water at a temperature range of 80 to $90^{\circ} \mathrm{C}$. After filtration, the obtained extract was evaporated under reduced pressure. The moisture content of the extract was about $40 \mathrm{w} / \mathrm{w} \%$. To prepare the Lo Han Kuo glycoside, Lo Han Kuo extract in paste form was diluted and the sweet components were selectively adsorbed onto a reversal phase column. Using an ethanolic solution, the sweet components that had been adsorbed onto the columns were eluted. Ethanol was then removed. Afterwards, spray drying was used to change the Lo Han Kuo fruit glycosides from a liquid state into powder form. The concentration of mogroside $\mathrm{V}$ was about $31 \mathrm{w} \%$.

Drugs The reagents used in the experiments were obtained from the sources shown in parentheses: histamine dihydrochloride (Nacalai Tesque, Kyoto), compound 48/80 (Sigma, St. Louis, MO, U.S.A.). Histamine and compound 48/80 were dissolved in physiological saline and administered intranasally and intradermally, respectively.

Nasal Rubbing Behavior To evaluate nasal rubbing, each time the animal rubbed or touched the area near the nose with its forepaws, it was counted as one event. Touches around the eyes and the mouth were disregarded. Before the experiment, the animals were placed into an observation cage $(32 \times 22 \times 10 \mathrm{~cm})$ for about $10 \mathrm{~min}$ for acclimatization. The extract and glycoside of Lo Han Kuo were administered orally. One hour later, $1 \mu \mathrm{l}$ of histamine $(5 \mathrm{nmol})$ was instilled into the bilateral nasal cavities. The animals were placed into the observation cage (one animal/cage), and nasal 
rubbing was counted for $60 \mathrm{~min}$.

Scratching Behavior Lo Han Kuo was orally administered to the animals. One hour later, $10 \mu \mathrm{g} / 0.02 \mathrm{ml}$ of compound 48/80 was intradermally injected into the rostral part of the shaved back of the mice. Immediately after injection, the animals were put into an observation cage $(11 \mathrm{~cm}$ in diameter) of MicroAct (Neuroscience, Tokyo), which detects and evaluates mouse-scratching behavior automatically and objectively. ${ }^{12)}$ Scratching behavior was observed for $60 \mathrm{~min}$.

Histamine Release from Mast Cells Peritoneal mast cells were harvested and purified by percoll density gradient centrifugation. ${ }^{13)}$ The collected mast cells were then incubated with physiological buffer solution (in mM: $\mathrm{NaCl} 140$, $\mathrm{CaCl}_{2}$ 0.9, glucose 5.6, HEPES 5, bovine serum albumin $0.05 \%$; pH 7.4, phosphate buffer solution) for $10 \mathrm{~min}$. Mast cell degranulation was induced by the addition of compound $48 / 80(5 \mu \mathrm{g} / 10 \mu \mathrm{l})$ before adding the samples. Lo Han Kuo extract and glycoside were added to each tube. The reaction was stopped $10 \mathrm{~min}$ later by cooling the tubes in ice water, and the tubes were centrifuged at $200 \times \boldsymbol{g}$ for $15 \mathrm{~min}$. Histamine content was measured in the supernatant and precipitate using an autoanalyzer. ${ }^{14)}$

Statistical Analysis All values are expressed as means standard errors of the mean (S.E.M.). Statistical evaluation of the results was performed by one-way analysis of variance (ANOVA) followed by Bonferroni test. A probability value of less than 0.05 was considered statistically significant.

\section{RESULTS}

Nasal Rubbing Behavior Induced by Histamine Fig-
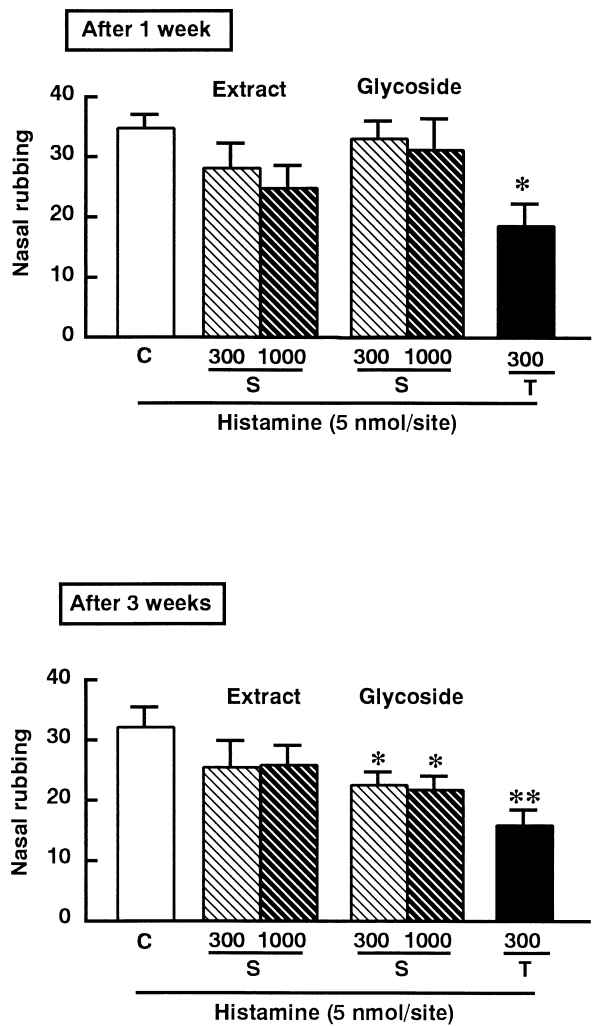

ure 1 shows the effects of a single administration of Lo Han Kuo extract and glycoside on histamine-induced nasal rubbing behavior. When histamine was administered into the nostrils of the mice, nasal rubbing was observed to an extent of $28.9 \pm 3.7$ times per $30 \mathrm{~min}$. The peak was observed $10 \mathrm{~min}$ after histamine instillation $(19.0 \pm 2.2)$, decreased to half of its value during the next $10 \mathrm{~min}(8.1 \pm 1.4)$, and almost disappeared during the last $10 \mathrm{~min}(1.7 \pm 0.7)$. Both the extract and glycoside caused no inhibitory effect, even at a dose of $1000 \mathrm{mg} / \mathrm{kg}$ by single administration. Tranilast, used as a positive control at a dose of $300 \mathrm{mg} / \mathrm{kg}$, also failed to inhibit histamine-induced nasal rubbing behavior. When the extract and glycoside were repeatedly administered once a day for 4 weeks, an inhibition of nasal rubbing behavior by Lo Han Kuo was gradually enhanced. The extract at doses of 300 and $1000 \mathrm{mg} / \mathrm{kg}$ caused a significant inhibition on hista-

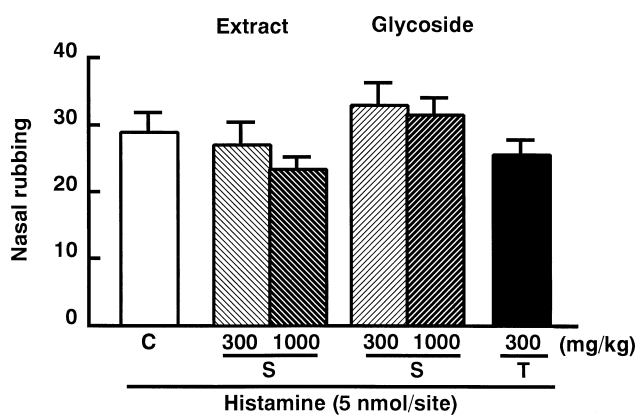

Fig. 1. The Effects of a Single Administration of Lo Han Kuo Extract and Glycoside on Histamine ( $5 \mathrm{nmol} /$ site)-Induced Nasal Rubbing in ICR Mice

Nasal rubbing was observed for $30 \mathrm{~min}$. Each column and vertical bar shows the means \pm S.E.M. $(n=15) . \mathrm{C}=$ control, $\mathrm{S}=$ Siraitia grosvenori, $\mathrm{T}=$ Tranilast.
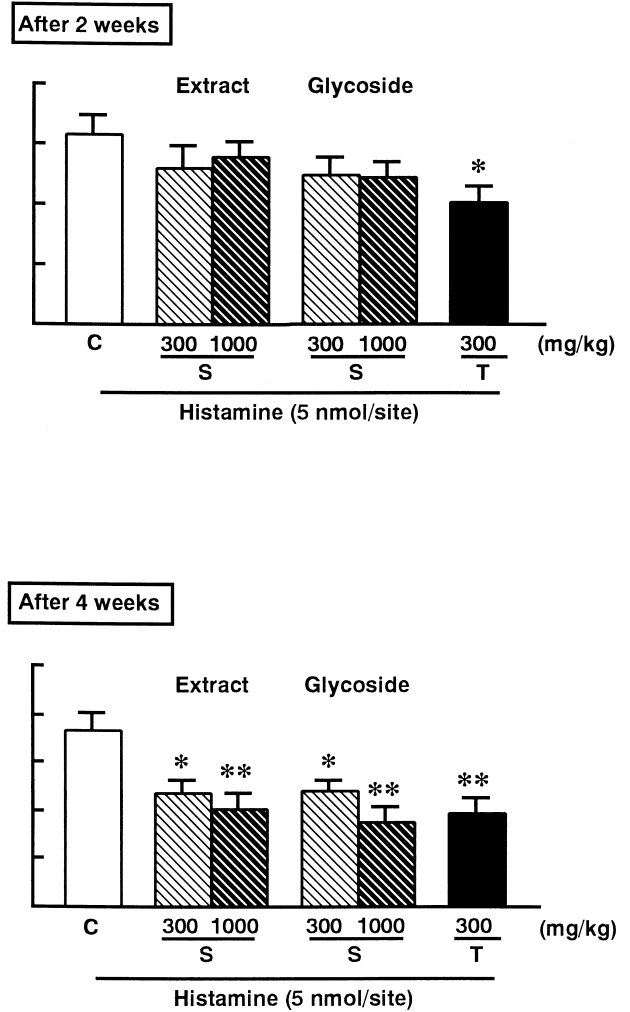

Fig. 2. The Effects of Consecutive Administration of Lo Han Kuo Extract and Glycoside on Histamine (5 nmol/site)-Induced Nasal Rubbing in ICR Mice Lo Han Kuo was administered daily for 4 weeks, and nasal rubbing was observed every week for 30 min. Each column and vertical bar shows the means \pm S.E.M. $(n=15)$. $*, * *$ Significantly different from the control group at $p<0.05$ and $p<0.01$, respectively. $\mathrm{C}=$ control, $\mathrm{S}=$ Siraitia grosvenori, $\mathrm{T}=$ Tranilast. 
mine-induced nasal rubbing after 4 weeks of repeated administration. Glycoside at doses of 300 and $1000 \mathrm{mg} / \mathrm{kg}$ also showed a significant inhibition of histamine-induced nasal rubbing after 3 and 4 weeks by repeated administration. In the case of tranilast, significant inhibitory effects were observed after 1 week of consecutive administration (Fig. 2).

Scratching Behavior Induced by Compound 48/80 Figure 3 shows the effects of a single administration of Lo Han Kuo on compound 48/80-induced scratching behavior. Both the extract and glycoside of Lo Han Kuo caused no significant effect at doses of 300 and $1000 \mathrm{mg} / \mathrm{kg}$. On the other hand, tranilast at a dose of $300 \mathrm{mg} / \mathrm{kg}$ significantly inhibited compound 48/80-induced scratching behavior. However, repeated administration of Lo Han Kuo for 4 weeks showed a

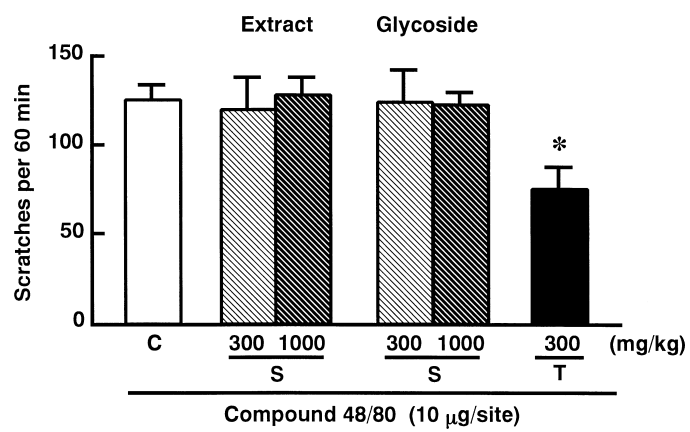

Fig. 3. The Effects of a Single Administration of Lo Han Kuo Extract and Glycoside on Compound 48/80 (10 $\mu \mathrm{g} /$ site)-Induced Scratching Behavior in ICR Mice

Each column and vertical bar shows the means \pm S.E.M. $(n=9)$. $*$ Significantly different from control group at $p<0.05 . \mathrm{C}=$ control, $\mathrm{S}=$ Siraitia grosvenori, $\mathrm{T}=$ Tranilast
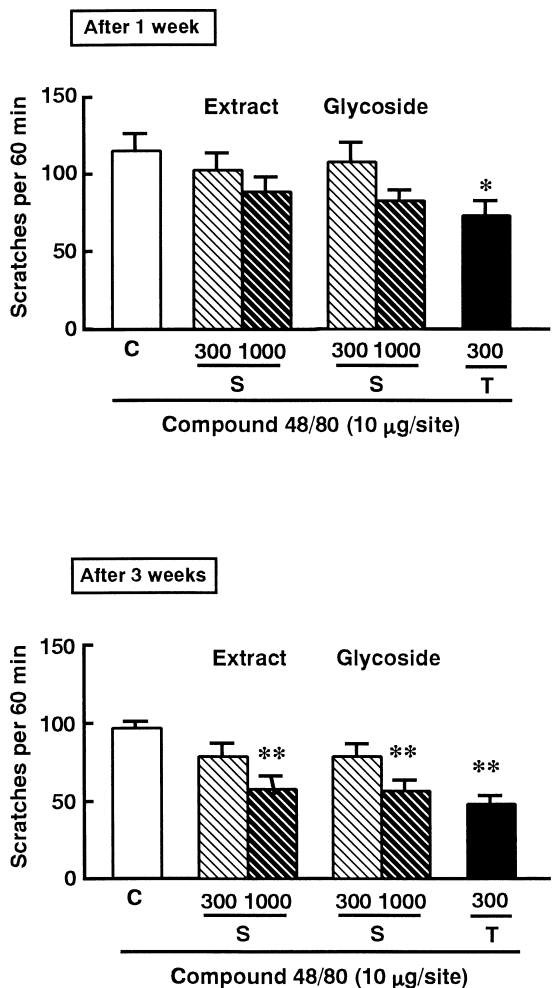

gradual inhibition of the itch response. The extract at a dose of $1000 \mathrm{mg} / \mathrm{kg}$ caused a significant inhibition by repeated administration for 2 weeks, and at a dose of $300 \mathrm{mg} / \mathrm{kg}$ it also caused significant inhibition by repeated administration for 4 weeks. After 3 weeks, glycoside at a dose of $1000 \mathrm{mg} / \mathrm{kg}$ started showing significant inhibitory effects, and after 4 weeks, at a dose of $300 \mathrm{mg} / \mathrm{kg}$, also caused a significant inhibition of compound 48/80-induced scratching behavior (Fig. 4).

Histamine Release from Rat Mast Cells Figure 5 shows the effects of the extract and glycoside of Lo Han Kuo on histamine release from rat peritoneal mast cells induced by compound $48 / 80$. Spontaneous histamine release was $6.7 \pm 0.4 \%(n=10)$, whereas the addition of compound $48 / 80$ at a concentration of $0.5 \mu \mathrm{g} / \mathrm{ml}$ induced a histamine release of $37.4 \pm 2.7 \%(n=10)$. Administration of both the extract and glycoside of Lo Han Kuo resulted in the concentrationrelated inhibition of this response. A concentration of $100 \mu \mathrm{g} / \mathrm{ml}$ of extract of Lo Han Kuo caused no significant inhibition; however, 300 and $1000 \mu \mathrm{g} / \mathrm{ml}$ significantly inhibited compound 48/80-induced histamine release. Almost similar findings were observed with the glycoside of Lo Han Kuo.

\section{DISCUSSION}

It is widely accepted that histamine derived from skin mast cells in response to various stimuli is an important mediator of itching in humans. Several in vitro as well as in vivo studies have confirmed that compound $48 / 80$ potently releases histamine from mast cells, ${ }^{15-17)}$ triggering skin responses
After 2 weeks

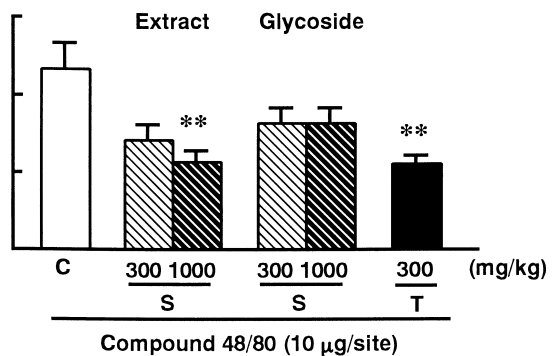

After 4 weeks

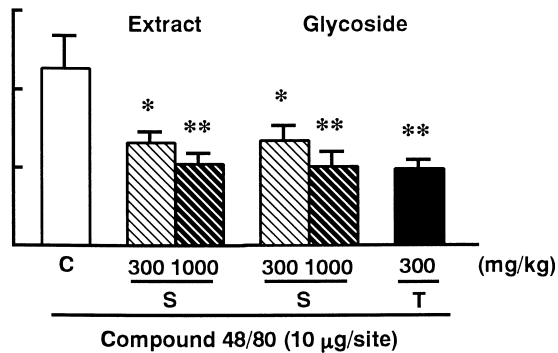

Fig. 4. The Effects of Consecutive Administration of Lo Han Kuo Extract and Glycoside on Compound 48/80 (10 $\mu \mathrm{g} /$ site)-Induced Scratching Behavior in ICR Mice

Lo Han Kuo was administered daily for 4 weeks, and scratching behavior was observed every week. Each column and vertical bar shows the means \pm S.E.M. $(n=9)$. $*, * *$ Significantly different from the control group at $p<0.05$ and $p<0.01$, respectively. $\mathrm{C}=$ control, $\mathrm{S}=$ Siraitia grosvenori, $\mathrm{T}=$ Tranilast. 


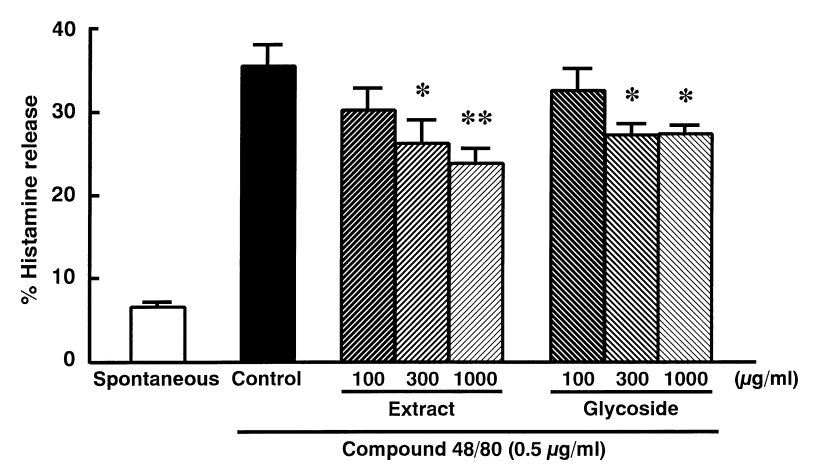

Fig. 5. The Effects of Lo Han Kuo Extract and Glycoside on Histamine Release from Rat Peritoneal Mast Cells Induced by Compound 48/80 $(0.5 \mu \mathrm{g} / \mathrm{ml})$

Each column and vertical bar shows the means \pm S.E.M. $(n=8)$. *,** Significantly different from the control group at $p<0.05$ and $p<0.01$, respectively.

such as scratching behavior or increased vascular permeability. In this study, histamine and compound 48/80 were used to induce nasal rubbing and scratching behavior, respectively, in ICR mice, responses that were significantly inhibited by consecutive pretreatment of the animals with Lo Han Kuo extract and glycoside. Nasal rubbing was induced by the administration of exogenous histamine, due to the fact that mucosal mast cells present in the nasal cavity are more sensitive to histamine than to compound $48 / 80$, as previously reported. ${ }^{18)}$ A single administration of Lo Han Kuo extract, glycoside or tranilast could block the nasal rubbing induced by histamine, suggesting that none of the compounds tested showed an $\mathrm{H}_{1}$ receptor antagonizing effect. Tranilast, an antiallergic drug that inhibits the release of chemical mediators from mast cells, has been reported to reduce the production of the major eosinophil-activating cytokine, IL-5, by daily administration. ${ }^{19)}$ There is also evidence that the application of histamine into the nasal mucosa induces the release of substance $P$ from peripheral terminals of the trigeminal nerve, ${ }^{20)}$ and that substance $\mathrm{P}$ releases histamine from nasal mucosa mast cells of subjects with or without allergic rhinitis. $^{21)}$ Although it is not clear, the inhibitory effect of tranilast may be occurring through blockage of these pathways. In the case of compound 48/80-induced scratching behavior, tranilast clearly blocked this response by a single administration via mast cell stabilizing effects, results that were not obtained by a single administration of Lo Han Kuo extract or glycoside. In general, natural products exert a stronger effect by consecutive administration ${ }^{17,22)}$; therefore, we also studied the effect of Lo Han Kuo administration for 4 weeks on both histamine-induced nasal rubbing and compound 48/80-induced scratching behavior. The inhibition of both nasal rubbing and scratching behavior by Lo Han Kuo was gradually enhanced by repeated administration.

To clarify the mechanism of action of Lo Han Kuo, we studied the effects of the extract and glycoside on histamine release using rat peritoneal mast cells. Lo Han Kuo extract and glycoside at $300 \mu \mathrm{g} / \mathrm{ml}$ or more significantly inhibited histamine release from rat peritoneal mast cells. Thus, we assumed that the inhibitory effects of nasal rubbing and scratching behavior may be attributable to the inhibition of mast cell degranulation. Sweet elements of Siraitia grosvenori have been proven to inhibit the oxidation of LDL. ${ }^{7)}$ Studies related to the carcinogenic activity of natural sweeteners isolated from Lo Han Kuo demonstrate that mogroside $\mathrm{V}$ and 11-oxo-mogroside $\mathrm{V}$ show an inhibition of peroxynitrite initiated and 12-o-tetradecanoylphorbol-13-acetate promoted tumors, through antioxidant properties. ${ }^{23}$ ) On the other hand, recent reports provide evidence that oxidative states and free radical generation play important roles in mast cell activation and degranulation. ${ }^{24,25)}$ Taken together, all these findings lead us to assume that the inhibitory effects of Lo Han Kuo on nasal rubbing and scratching behavior may be due to an inhibition of histamine release from mast cells through the prevention of superoxide anion generation.

\section{REFERENCES}

1) Kasai R., Nie R. L., Nashi K., Ohtani K., Zhou J., Tao G. D., Tanaka O., Agric. Biol. Chem., 53, 3347-3349 (1989).

2) Konoshima T., Takasaki M., Pure Appl. Chem., 74, 1309-1316 (2002).

3) Matsumoto K., Kasai R., Ohtani K., Tanaka O., Chem. Pharm. Bull., 38, 2030-2032 (1990).

4) Sekiya J., Foods \& Food Ingredients Journal of Japan, 162, 24-28 (1994).

5) Maeda H., Akaike T., Biochemistry, 63, 854-865 (1998).

6) Felley-Bosco E., Cancer Metastasis Rev., 17, 25-37 (1998).

7) Takeo E., Yoshida H., Tada N., Shingu T., Matsuura H., Murata Y., Yoshikawa S., Ishikawa T., Nakamura H., Ohsuzu F., Kohda H., J. Atheroscler. Thromb., 9, 114-120 (2002).

8) Shi H., Hiramatsu M., Komatsu M., Kayama T., Biochem. Mol. Biol. Int., 40, 1111-1121 (1996).

9) Fukuishi N., Sakaguchi M., Matsuura S., Nakagawa C., Akagi R., Akagi M., Biochem. Mol. Med., 61, 107-113 (1997).

10) Fukuishi N., Kan T., Hirose K., Akagi R., Akagi M., Jpn. J. Pharmacol., 68, 449-452 (1995).

11) Yoshimaru T., Suzuki Y., Matsui T., Yamashita K., Ochiai T., Yamaki M., Shimizu K., Clin. Exp. Allergy, 32, 612-618 (2002).

12) Inagaki N., Igeta K., Kim J. F., Nagao M., Shiraishi N., Nakamura N., Nagai H., Eur. J. Pharmacol., 448, 175-183 (2002).

13) Németh A., Röhlich P., Europ. J. Cell. Biol., 20, $272-275$ (1980).

14) Siraganian R. P., Anal. Biochem., 57, 383-394 (1974).

15) Nakayama Y., Mio M., Sugimoto Y., Fujii Y., Kamei C., Methods Find. Exp. Clin. Pharmacol., 24, 267-273 (2002).

16) Kuraishi Y., Nagasawa T., Hayashi K., Satoh M., Eur. J. Pharmacol., 257, 229-233 (1995).

17) Shinmei Y., Hossen M. A., Okihara K., Sugimoto H., Yamada H., Kamei C., Int. Immunopharmacol., 4, 1431-1436 (2004)

18) Kayasuga R., Sugimoto Y., Watanabe T., Kamei C., Int. Immunopharmacol., 2, 745-750 (2002).

19) Hiratochi M., Takamoto M., Tatemichi S., Sugane K., Int. J. Immunopharmacol., 22, 463-471 (2000).

20) Tani E., Senba E., Kokumai S., Masuyama K., Ishikawa T., Tohyama M., Neurosci. Lett., 112, 1-6 (1990).

21) Hanf G., Schierhorn K., Brunnee T., Noga O., Verges D., Kunkel G., Inflamm. Res., 49, 520-523 (2000).

22) Inoue T., Sugimoto Y., Masuda H., Kamei C., Biol. Pharm. Bull., 24, 92-95 (2001).

23) Takasaki M., Konoshima T., Murata Y., Sugiura M., Nishino H., Tokuda H., Matsumoto K., Kasai R., Yamasaki K., Cancer Lett., 198, $37-42$ (2003).

24) Fukuishi N., Takahashi H., Harada N., Kanoh R., Matsui N., Akagi M., Int. Arch. Allergy Immunol., 126, 140-146 (2001).

25) Kanoh R., Hatano T., Ito H., Yoshida T., Akagi M., Phytomedicine, 7 , 297-302 (2000) 